\title{
Abstract \\ Dynamics of Anisotropic Cylindrical Collapse in Energy-Momentum Squared Gravity ${ }^{\dagger}$
}

\author{
Muhammad Sharif *(D) and Muhammad Zeeshan Gul
}

check for

updates

Citation: Sharif, M.; Gul, M.Z. Dynamics of Anisotropic Cylindrical Collapse in Energy-Momentum Squared Gravity Phys. Sci. Forum 2021, 2, 40. https://doi.org/10.3390/ ECU2021-09513

Academic Editor: Banibrata

Mukhopadhyay

Published: 19 March 2021

Publisher's Note: MDPI stays neutral with regard to jurisdictional claims in published maps and institutional affiliations.

Copyright: (c) 2021 by the authors. Licensee MDPI, Basel, Switzerland. This article is an open access article distributed under the terms and conditions of the Creative Commons Attribution (CC BY) license (https:// creativecommons.org/licenses/by/ $4.0 /)$.
Department of Mathematics, University of the Punjab, Lahore-54590, Pakistan; mzeeshangul.math@gmail.com

* Correspondence: msharif.math@pu.edu.pk

+ Presented at the 1st Electronic Conference on Universe, 22-28 February 2021; Available online: https:/ / ecu2021.sciforum.net/.

\begin{abstract}
This paper deals with the dynamics of cylindrical collapse with anisotropic matter configuration in the context of energy-momentum squared gravity. This covariant generalization of general relativity allows the presence of $\mathrm{T}_{-} \mathrm{abT} \mathrm{T}^{\wedge} \mathrm{ab}$ in the action of functional theory. Consequently, the relevant field equations are different from general relativity only in the presence of matter sources. In this theory, there is a maximum energy density and a minimum scale factor of the early universe. This means that there is a bounce at early times which avoids the presence of an early-time singularity. Moreover, this theory possesses a true sequence of cosmological eras. However, the cosmological constant does not play an important role in the early times and becomes important only after the matter-dominated era. In this theory, the "repulsive" nature of the cosmological constant plays a crucial role at early times in resolving the singularity. We formulate the corresponding field equations as well as junction conditions. We construct dynamical equations through the Misner-Sharp technique and examine the impact of energy-momentum squared gravity on the collapse rate. We develop a relation among fluid parameters, correction terms and Weyl scalar and examine the effects of anisotropy, effective matter variables and correction terms on the collapsing phenomenon. Due to the presence of anisotropic pressure, spacetime is no longer considered to be conformally flat. To obtain conformally flat spacetime, we neglect the impact of anisotropy and assume the isotropic matter distribution which yields homogeneity of the energy density and conformally flat spacetime. The hydrodynamical force determines the stability of the system and prevents the collapsing as well as expanding process for the constant energy-momentum squared gravity model. We conclude that positive correction terms and anisotropy provide the anti-gravitational behavior leading to the stability of self-gravitating objects and hence prevent the collapsing process.
\end{abstract}

Keywords: energy-momentum squared gravity; gravitational collapse; anisotropy; dynamical equations

Supplementary Materials: The supplementary file is available online at https:/ / www.mdpi.com/ article/10.3390/ECU2021-09513/s1.

Institutional Review Board Statement: Not applicable.

Informed Consent Statement: Not applicable.

Data Availability Statement: No data has been used. 